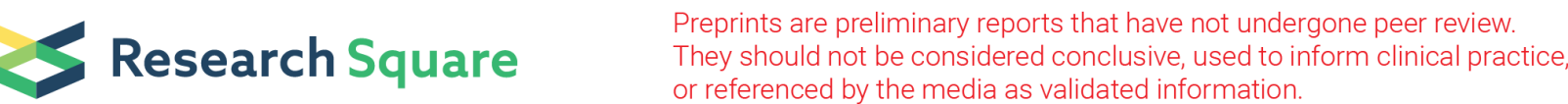

\section{Efficacy and safety of CT-P59 plus standard of care: a phase 2/3 randomized, double-blind, placebo- controlled trial in outpatients with mild-to-moderate SARS-CoV-2 infection}

Joong Sik Eom ( $\nabla$ helppl@gilhospital.com )

Gachon University Gil Medical Center

Michael Ison

Northwestern University

Anca Streinu-Cercel

Carol Davila University of Medicine and Pharmacy

Oana Săndulescu

Carol Davila University of Medicine and Pharmacy

Liliana-Lucia Preotescu

Carol Davila University of Medicine and Pharmacy

Yeon-Sook Kim

Chungnam National University

Jin Yong Kim

Incheon Medical Center

Shin Hyea Cheon

Chungnam National University School of Medicine

Young Rock Jang

Incheon Medical Center

\section{Sang Joon Lee}

Celltrion Inc

Sung Hyun Kim

Celltrion Inc

llsung Chang

Celltrion Inc

Jee Hye Suh

Celltrion Inc

Seul Gi Lee

Celltrion Inc

Mi Rim Kim

Celltrion Inc 
Da Rae Chung

Celltrion Inc https://orcid.org/0000-0002-4399-8945

Han Na Kim

Celltrion Inc

Adrian Streinu-Cercel

Carol Davila University of Medicine and Pharmacy

\section{Article}

Keywords: Monoclonal Antibody, Hospitalization Prevention, Clinical Recovery, Treatment-emergent Adverse Events

Posted Date: March 15th, 2021

DOl: https://doi.org/10.21203/rs.3.rs-296518/v1

License: (c) (i) This work is licensed under a Creative Commons Attribution 4.0 International License. Read Full License 


\section{Abstract}

CT-P59, a monoclonal antibody with potent neutralizing activity against severe acute respiratory syndrome coronavirus 2 , may ameliorate symptoms and prevent hospitalization in outpatients with mildto-moderate disease. We report findings from part one of a two-part randomized, placebo-controlled, double-blind study (NCT04602000; EudraCT: 2020-003369-20). Outpatients with mild-to-moderate COVID19 received a single dose of CT-P59 $40 \mathrm{mg} / \mathrm{kg}(n=101)$, CT-P59 $80 \mathrm{mg} / \mathrm{kg}(n=103)$, or placebo $(n=103)$. Median (95\% confidence interval [CI]) time to conversion to negative RT-qPCR result (coprimary endpoint) was 12.8 days (9.00-12.84) with CT-P59 $40 \mathrm{mg} / \mathrm{kg}, 11.9$ days (8.94-12.91) with CT-P59 $80 \mathrm{mg} / \mathrm{kg}$, and 12.9 days $(12.75-13.99)$ with placebo. Median $(95 \% \mathrm{Cl})$ time to clinical recovery (coprimary endpoint) was 5.4 days (3.97-6.78) with CT-P59 $40 \mathrm{mg} / \mathrm{kg}, 6.2$ days (5.53-7.85) with CT-P59 $80 \mathrm{mg} / \mathrm{kg}$, and 8.8 days $(6.72-11.73)$ with placebo. The proportion $(95 \% \mathrm{Cl})$ of patients requiring hospitalization or oxygen therapy was lower with CT-P59 $40 \mathrm{mg} / \mathrm{kg}(4.0 \%$ [1.6-9.7\%]) and CT-P59 $80 \mathrm{mg} / \mathrm{kg}(4.9 \%$ [2.1-10.9\%]) versus placebo (8.7\% [4.7-15.8\%). CT-P59 was well tolerated and no serious treatment-emergent adverse events or deaths occurred. In summary, CT-P59 accelerated viral and clinical recovery from COVID-19 and was well tolerated in patients with mild-to-moderate infection.

\section{Introduction}

The severe acute respiratory syndrome coronavirus 2 (SARS-CoV-2) pandemic represents an ongoing global health crisis of unprecedented scale ${ }^{1-3}$. While most patients experience asymptomatic or mild-tomoderate disease, some progress to experience more severe symptoms and may require hospitalization ${ }^{4,5}$.

Effective treatments are urgently needed to reduce SARS-CoV-2 infection-related morbidity and mortality, and to reduce the associated burden on public health services ${ }^{6}$. Therapies that can be applied early in the course of clinical disease can speed clinical recovery, shorten the duration of viral shedding, and reduce the need for hospitalization, with a resultant reduction of the burden on the overall healthcare system. Anti-SARS-CoV-2 spike (S) antibodies, which target cellular entry of SARS-CoV-2 through interaction with the angiotensin-converting enzyme 2 (ACE2) receptor ${ }^{7-11}$, have been demonstrated to reduce hospitalizations, viral titers, and clinical symptoms in patients with SARS-CoV-2 infection ${ }^{12-14}$; the therapy has also been shown to reduce the risk of infection with SARS-CoV-2 in nursing home residents ${ }^{15,16}$.

CT-P59 is a potent neutralizing antibody against various SARS-CoV-2 isolates, including the D614G S protein variant ${ }^{17}$. Complex crystal structure studies of the CT-P59 Fab/SARS-CoV-2 receptor binding domain (RBD) suggest that CT-P59 blocks the interaction regions of the SARS-CoV-2 S protein RBD for ACE2 ${ }^{17}$. Administration of CT-P59 was shown to reduce viral load and alleviate clinical symptoms in animal models of SARS-CoV-2 infection ${ }^{17}$. CT-P59 has also exhibited a promising safety profile in healthy volunteers and patients with mild SARS-CoV-2 infection, and potential antiviral and clinical efficacy has been observed in patients with mild SARS-CoV-2 symptoms ${ }^{18}$. Based on these findings, along with the 
results reported in this manuscript, CT-P59 was granted conditional marketing authorization by the South Korean Ministry of Food and Drug Safety (MFDS) and an application has been submitted to the European Medicines Agency (EMA) for marketing authorization.

We report 28-day results from part 1 of a two-part phase 2/3 study of CT-P59 in outpatients with mild-tomoderate SARS-CoV-2 infection.

\section{Results}

Patient disposition and baseline characteristics. Patient enrolment began on 07 October 2020 and the last patient's Day 28 visit was on 18 December 2020. Screening was conducted at 23 centers across South Korea, Romania, Spain and USA. A total of 371 patients were screened and 327 were randomized and included in the intent-to-treat (ITT) population (CT-P59 $40 \mathrm{mg} / \mathrm{kg}: n=105$; CT-P59 $80 \mathrm{mg} / \mathrm{kg}: n=111$; placebo: $n=111$; Fig. 1). Eight patients (2.4\%) discontinued the study during the treatment period (CT-P59 $40 \mathrm{mg} / \mathrm{kg}: n=3$; CT-P59 $80 \mathrm{mg} / \mathrm{kg}: n=3$; placebo: $n=2$ ). There was one major protocol deviation: one patient randomized to the placebo group had instead partially received CT-P59; this patient was considered as having received CT-P59 $40 \mathrm{mg} / \mathrm{kg}$ and was included in the CT-P59 $40 \mathrm{mg} / \mathrm{kg}$ treatment group for the efficacy and safety analyses, but was excluded from the PK analyses.

In the ITT population, participants were white (87.5\%) or Asian (12.5\%) and $49.2 \%$ were female; median age was 51.0 years (interquartile range: $41-60$ ) and mean body mass index was $27.0 \mathrm{~kg} / \mathrm{m}^{2}$ (standard deviation: 4.4). Moderate disease was reported in 189 patients (57.8\%). Baseline demographic and disease characteristics were generally well balanced between groups (Table 1). Concomitant medications are listed in Supplementary Table 1.

Twenty patients in the ITT population did not have confirmed SARS-CoV-2 infection by RT-qPCR at day 1 or both day 1 and day 2, and were excluded from the ITTI population ( $N=307$ patients [CT-P59 $40 \mathrm{mg} / \mathrm{kg}$ : $n=101$; CT-P59 80 mg/kg: $n=103$; placebo: $n=103]$, see Supplementary Table 2).

Efficacy (ITTI population). The median time $(95 \% \mathrm{Cl})$ to conversion to negative RT-qPCR (threshold $<2.33 \log _{10}$ copies/ml) up to day 28 was numerically shorter in the CT-P59 $40 \mathrm{mg} / \mathrm{kg}$ and $80 \mathrm{mg} / \mathrm{kg}$ groups than in the placebo group (median: 12.8 and 11.9 days vs 12.9 days, respectively) and in the combined CT-P59 versus placebo groups (median: 12.7 vs 12.9 days) (Table 2; Fig. 2a). The corresponding improvement rate ratios (with $95 \% \mathrm{Cls}$ ) were $1.346(1.001-1.810 ; P=0.048), 1.215(0.90-$ 1.63; $P=0.198)$, and $1.275(0.99-1.65 ; P=0.063)$ for the CT-P59 $40 \mathrm{mg} / \mathrm{kg}, 80 \mathrm{mg} / \mathrm{kg}$, and combined groups, relative to placebo (Table 2). When a threshold of $<3.0 \log _{10} \mathrm{copies} / \mathrm{ml}$ was applied in a post-hoc analysis CT-P59 further reduced median time to conversion to negative RT-qPCR (6.0 days [combined CTP59] vs 8.9 days [placebo]; Table 2 and Supplementary Fig. 1).

The proportion of patients achieving conversion to negative RT-qPCR was higher in the CT-P59 groups than in the placebo group up to days 14 and 28 (Table 2). Greater reductions from baseline in viral load were observed in the CT-P59 groups than in the placebo group. A $3-\log _{10}$ copies/ml reduction in viral load 
was achieved with CT-P59 between baseline and day 7; a similar reduction was not reached in the placebo group until day 10 (Supplementary Fig. 2).

The median $(95 \% \mathrm{Cl})$ time to clinical recovery up to day 14 was shorter in the CT-P59 combined group versus the placebo group (5.7 [5.15-7.00] vs 8.8 [6.72-11.73] days, respectively; Table 2 and Fig. $2 b$ ). Clinical recovery ratios (with 95\% Cis) were 1.562 (1.11-2.20; $P=0.010), 1.429(1.02-2.01 ; P=0.039)$, and $1.489(1.11-2.01 ; P=0.008)$ in the CT-P59 $40 \mathrm{mg} / \mathrm{kg}, 80 \mathrm{mg} / \mathrm{kg}$, and combined groups, respectively (Table 2). Clinical recovery ratios also favored CT-P59 over placebo in prespecified subgroups by disease status (Table 2). Clinical recovery rate ratios (with $95 \% \mathrm{Cis}$ ) for the CT-P59 combined group versus placebo group were 1.615 (1.05-2.49) in patients with mild SARS-CoV-2 infection (median 4.8 vs 6.9 days), 1.511 (0.99-2.30) in patients with moderate infection (median 6.5 vs 10.8 days), and $1.545(0.90-2.65)$ in patients aged $\geq 50$ years with moderate infection (median 6.8 vs 13.0 days).

The proportion of patients with clinical symptoms requiring hospitalization or oxygen therapy due to SARS-CoV-2 infection was significantly lower in the CT-P59 groups than in the placebo group (CT-P59 40 mg/kg: 4 [4.0\%]; CT-P59 80 mg/kg: 5 [4.9\%]; placebo: 9 [8.7\%]; Fig. 2c and Table 2). Notably, only patients with moderate SARS-CoV-2 infection contributed events to this composite endpoint; the proportions of patients meeting this endpoint in the CT-P59 combined group was $>50 \%$ lower than in the placebo group among patients with moderate infection ( $7.2 \%$ vs $15.8 \%$, respectively) and in patients aged $\geq 50$ years with moderate infection ( $8.8 \%$ vs $23.7 \%$, respectively). CT-P59 also effectively reduced the proportion of patients reporting the requirement for supplemental oxygen, hospital admission, and rescue therapy, individually, and no all-cause mortality was reported up to day 28 (Table 2).

Safety. Overall, 182 TEAEs were reported in 92 patients (28.3\%). A similar proportion of patients experienced $\geq 1$ TEAE across treatment groups (Table 3 ) and the majority of TEAEs were CTCAE grade 1 or 2 in intensity. The most frequently reported TEAE considered related to study drug was hypertriglyceridemia in patients receiving CT-P59 $40 \mathrm{mg} / \mathrm{kg}$ (3 patients [2.9\%]) and infusion-related reaction or hypertriglyceridemia in patients receiving placebo (2 [1.8\%] patients each), noting that except for those in the PK substudy, participants were not required to fast before administration of study drug. No TEAE considered to be related to study drug was reported in >1 patient in the CT-P59 $80 \mathrm{mg} / \mathrm{kg}$ group. There were no TESAEs or TEAEs leading to permanent study discontinuation, and infusion-related reactions were reported in a low number of patients (CT-P59 40 mg/kg: 1 [1.0\%]; CT-P59 80 mg/kg: 0; placebo: $2[1.8 \%])$.

Generally, there were no notable differences between groups in terms of changes from baseline in laboratory parameters (hematology and clinical chemistry), vital signs, or ECG results. The proportion of patients positive for ADAs was low in the CT-P59 groups (day 28 values were: CT-P59 $40 \mathrm{mg} / \mathrm{kg}$ : 0; CTP59 80 mg/kg: 3 [2.7\%]; placebo: 5 [4.5\%]). No antibody-dependent enhancement events were reported up to day 28.

Pharmacokinetics. The mean CT-P59 serum concentration was higher in the CT-P59 $80 \mathrm{mg} / \mathrm{kg}$ group than in the $40 \mathrm{mg} / \mathrm{kg}$ group at all time points following intravenous infusion (Supplementary Fig. 3). 
Serology. The proportions of patients with immunoglobulin (Ig) M or IgG positivity increased over time and was similar across groups at all time points (Supplementary Table 3).

\section{Discussion}

These data demonstrate that CT-P59 results in acceleration of clinical and viral improvement in outpatients with mild-to-moderate COVID-19. Additionally, CT-P59 reduced the need for hospitalization or oxygen therapy while being generally well tolerated.

CT-P59 was associated with a shorter time to conversion to a negative RT-qPCR result. While this analysis did not measure infectious virus, viral loads of $<4 \log _{10}$ copies $/ \mathrm{ml}$ are not expected to be detected by cell culture method ${ }^{19,20}$. As such, the reductions seen in patients treated with CT-P59 could also reduce the risk of transmission. Further studies would need to be performed to confirm this finding but would add greater support for the early use of monoclonal antibodies (mAbs) among ambulatory patients with SARS-CoV-2 infection. These data are also in line with other studies of mAbs that shorten the duration of viral shedding with SARS-CoV-2 ${ }^{12-14}$.

CT-P59 was well tolerated, no clinically significant safety issues were identified, and there were no deaths up to day 28 . Only one patient receiving CT-P59 $(40 \mathrm{mg} / \mathrm{kg})$ experienced an infusion-related reaction (Grade 2 pyrexia and Grade 1 dyspnea were experienced after the study drug was completely administered, but recovered on the same day after receiving paracetamol and oxygen therapy). CT-P59 did not appear to interfere with the formation of antibodies (no antibody-dependent enhancement events were reported), no ADAs were measured in the CT-P59 $40 \mathrm{mg} / \mathrm{kg}$ group, and the proportion of patients with ADAs in the CT-P59 $80 \mathrm{mg} / \mathrm{kg}$ group was $<3.0 \%$. Interestingly, ADAs were identified in $4.5 \%$ of patients in the placebo group; this is likely due to the presence of pre-existing antibodies, which are either part of the natural population of antibodies or part of the adaptive immune response to environmental antigens $^{21,22}$.

Despite having dose-dependent PK, no dose-response relationship was observed (data not shown). Based on these data, the second part of this study will only compare the $40 \mathrm{mg} / \mathrm{kg}$ dose with placebo in a larger population of patients with mild-to-moderate SARS-CoV-2 infection. Part 2 aims to confirm clinically meaningful therapeutic efficacy for CT-P59, as determined by the proportion of patients with clinical symptoms requiring hospitalization or oxygen therapy, or experiencing mortality, due to SARS-CoV-2 infection up to day 28.

To our knowledge, this study represents the fourth study to assess mAbs for the treatment of ambulatory adults. The published studies of bamlanivimab with or without etesevimab ${ }^{13,14}$, and of casirivimab and imdevimab ${ }^{12}$, much like the present study, have demonstrated improvement in clinical and virologic recovery, as well as reduction in the risk of hospitalization or medically attended visits. These clinically significant findings are confirmed in this study and suggest that early use of mAbs in patients with 
COVID-19 may reduce the burden on the healthcare system, onward transmission, and symptoms more quickly.

The current study enrolled few black or Hispanic patients, populations that are disproportionately affected by SARS-CoV-2 infection ${ }^{23,24}$. Likewise, there was a relatively small proportion $(20-30 \%)$ of elderly patients enrolled, another group that is at high risk of complications and death from COVID-1925. The impact of CT-P59 on infectious virus has not been studied but it would be important to assess this in order to inform treatment decisions to reduce the risk of transmission.

Based on these findings, we conclude that CT-P59 is well tolerated and has the potential to alleviate the substantial burden placed on healthcare systems by SARS-CoV-2 during the ongoing pandemic. On February 5, 2021, the South Korean MFDS granted conditional marketing authorization for the use of CTP59 (regdanvimab) in patients with mild SARS-CoV-2 infection who are aged $\geq 60$ years or who have at least one of the following underlying medical conditions (cardiovascular disease, chronic respiratory disease, hypertension, diabetes mellitus), as well as in patients with moderate SARS-CoV- 2 infection. The second part of the study is ongoing and will assess the impact of CT-P59 on clinical recovery and the complications of COVID-19.

\section{Methods}

Study design and oversight. This phase $2 / 3$, randomized, parallel-group, placebo-controlled, double-blind study (NCT04602000; EudraCT: 2020-003369-20) enrolled outpatients with mild-to-moderate SARS-CoV-2 infection. The disease was classified as mild or moderate at baseline based on guidance from the World Health Organization ${ }^{26}$ : a patient was considered to have moderate disease based on the presence of radiography-confirmed pneumonia, and to have mild disease based on the absence of radiographyconfirmed pneumonia at baseline. Part 1 of the study (reported to 28 days in the present manuscript) comprised three periods: screening (days -7 to day 1), treatment (day 1 to day 90 end-of-treatment [EOT] visit), and follow-up (EOT visit to day 180). Patients received a single infusion of study drug on day 1 . Part 2 of the study (ongoing) aims to confirm the effect of CT-P59 on clinical symptoms requiring hospitalization, oxygen therapy, or mortality due to SARS-CoV-2 infection, and will be reported separately.

The study was conducted in accordance with the ethical principles of the Declaration of Helsinki and in compliance with the International Council for Harmonisation Good Clinical Practice and applicable regulatory requirements. The protocol and all applicable amendments were reviewed and approved by local or national independent ethics committees prior to study initiation and the study was monitored by an independent data safety monitoring board. All participants provided written informed consent.

Participants. Eligible participants were aged $\geq 18$ years, diagnosed with SARS-CoV- 2 infection at the study centers at screening using the sponsor-supplied rapid SARS-CoV-2 diagnostic test (DiaTrust ${ }^{\mathrm{TM}}$, Celltrion, Inc., Incheon, Republic of Korea) or locally conducted reverse transcription polymerase chain reaction (RT-PCR), had an oxygen saturation of $>94 \%$ on room air, and did not require supplemental 
oxygen. Participants were required to have symptom onset (feverishness, cough, shortness of breath, sore throat, body/muscle pain, fatigue, headache, chills, nasal congestion, loss of taste or smell, or diarrhea) within 7 days before study drug administration. Patients with a current serious health condition or with ongoing or history of active or severe infections were excluded. Comprehensive eligibility criteria are provided in the protocol (see Supplementary Information).

Randomization and masking. Randomization was performed using an interactive web response system and a randomization schedule prepared by unblinded biostatisticians. Randomization was stratified by age ( $\geq 60$ vs $<60$ years), region (USA vs Asia vs EU vs other), baseline comorbidities (yes vs no for having at least one of cardiovascular disease, chronic respiratory disease, hypertension, diabetes mellitus, or pneumonia), and participation in the pharmacokinetic (PK) substudy (yes vs no). Participants, personnel, and outcome assessors were blinded to treatment allocation for the duration of the study. CT-P59 and placebo were supplied in identical vials identified by a study drug number. Designated unblinded personnel prepared the study drug for infusion.

Procedures. Participants were assigned randomly (1:1:1 ratio) to receive a single dose of CT-P59 40 $\mathrm{mg} / \mathrm{kg}$, CT-P59 $80 \mathrm{mg} / \mathrm{kg}$, or placebo. CT-P59 and placebo were reconstituted in $250 \mathrm{~mL}$ of $0.9 \%$ sodium chloride and administered via intravenous infusion over $90 \pm 15$ minutes. All patients received optimal standard-of-care treatment, including rehydration therapy, antipyretics, or antitussives at the investigator's discretion, but excluding antiviral drugs and/or possible SARS-CoV-2 active drugs (only to be administered as rescue therapies).

Nasopharyngeal swabs for assessment of viral shedding (based on quantitative RT-PCR [RT-qPCR]) were taken predose on day 1 , and at 24, 48, 72, 96, 120, 144, 216, 312 (day 10), 384 (day 17), 480 (day 21), and 648 (day 28) hours after study drug administration.

Participants were required to complete a patient diary on days $1-28$, which included a SARS-CoV-2 symptom checklist. The checklist, which was to be completed by all patients at screening and twice daily from days 1-28, included seven symptoms of SARS-CoV-2 infection (fever, cough, shortness of breath or difficulty breathing, sore throat, body pain or muscle pain, fatigue, and headache).

Disease status monitoring was performed throughout the study (e.g., requirement for supplemental oxygen, intensive care unit [ICU] transfer, mechanical ventilation use, hospitalization, and rescue therapy use).

Safety was assessed throughout the study based on the incidence, type, severity, and causality of treatment-emergent adverse events (TEAEs), treatment-emergent serious adverse events (TESAEs), and TEAEs of special interest. TEAEs were coded according to the Medical Dictionary for Regulatory Activities, Version 23.1, and graded for severity according to the Common Terminology Criteria for Adverse Events (CTCAE), Version 5.0. Hypersensitivity monitoring was performed before and after administration of study drug on day 1 . Vital signs measurements, physical examination findings (before study drug 
administration and EOT only), electrocardiogram (ECG) findings, and clinical laboratory analyses were assessed periodically throughout the study.

Details of the study drug formulation; SARS-CoV-2 symptom checklist; and PK, serology (including antidrug antibodies [ADAs]), and virology assessments are described in the Supplementary Information.

Study endpoints. The primary study endpoints were time to conversion to negative nasopharyngeal swab specimen based on RT-qPCR (negative titer threshold: $2.33 \log _{10}$ copies $/ \mathrm{ml}$ ) up to day 28 and time to clinical recovery up to day 14 .

Patients who had negative RT-qPCR results at two or more consecutive time points were considered as satisfying the criteria for conversion to negative nasopharyngeal swab specimen based on RT-qPCR result (the first of the two consecutive time points was taken as the time to conversion to negative RTqPCR result). Clinical recovery was defined as all symptom scores of "absent" or "mild" for $\geq 24$ hours based on checklist results; symptoms scored as moderate or severe at baseline were required to be scored as mild or absent at recovery, whereas symptoms rated as mild or absent at baseline were required to be rated as absent at recovery.

Secondary efficacy endpoints included the following: the proportion of patients with clinical symptoms requiring hospitalization ( $\geq 24$ hours of acute care), oxygen therapy ( $\geq 24$ hours of supplemental oxygen care, with oxygen saturation of $\leq 94 \%$ on room air prior to administration), or death due to due to SARSCoV-2 up to day 28; the proportion of patients with conversion to negative RT-qPCR result; and the proportions of patients with hospital admission requiring supplemental oxygen, with mechanical ventilation use, requiring rescue therapy, with ICU admission (individual endpoints; each due to SARSCoV-2 infection), or with all-cause mortality.

Primary endpoint definitions and a comprehensive list of efficacy, PK, and virology endpoints are described in the Supplementary Information.

Statistical analysis. It was estimated that a sample size of 100 individuals per group would provide at least $80 \%$ power at a two-sided significance level of 0.05 to detect an increase in the improvement rate ratio between CT-P59 groups and placebo for the primary endpoints.

Efficacy was assessed in the intent-to-treat infected (ITTI) population, which comprised all randomly assigned patients with confirmed SARS-CoV-2 infection assessed by pre-infusion RT-qPCR on day 1 and receiving a partial or complete dose of study drug. If the pre-infusion result on day 1 was confirmed negative or missing and the day 2 result was confirmed positive, the patient was also considered as having confirmed SARS-CoV-2 infection. The safety population included all randomized participants who received a partial or full dose of the study drug.

Primary efficacy analyses were performed using stratified log-rank test for time-to-event endpoints. Improvement/clinical recovery rate ratios (with 95\% confidence intervals [Cls]) were estimated using a 
stratified Cox proportional hazards model. Adjustments for multiple testing were not performed.

Secondary efficacy endpoints were summarized using descriptive statistics, frequency tables, or KaplanMeier (time-to-event) methods.

Subgroup analyses of the primary endpoints and one of the secondary efficacy endpoints were conducted according to SARS-CoV-2 severity (mild vs moderate; prespecified) and age ( $\geq 50$ years; exploratory). Post-hoc analyses of the primary efficacy outcome - time to conversion to negative RTqPCR - were conducted based on negative titer thresholds of 3.0 and $4.0 \log _{10}$ copies $/ \mathrm{ml}$.

All statistical analyses were conducted using Statistical Analysis System (SAS) software, Version 9.4 (SAS Institute Inc., Cary, NC, USA). Sample size calculation assumptions, definitions of additional analysis populations, and statistical methodologies (including missing data handling) are included in the statistical analysis plan (see Supplementary Information).

\section{Data availability}

The study protocol and statistical analysis plan are available online as supplementary information. Individual participant data cannot be made available.

\section{Declarations}

\section{Acknowledgements}

The authors thank all patients and investigators involved in the study. Medical writing support (including development of a draft outline and subsequent drafts in consultation with the authors, assembling tables and figures, collating author comments, copyediting, fact checking, and referencing) was provided by Duncan Campbell, PhD, CMPP, at Aspire Scientific (Bollington, UK), and was funded by Celltrion, Inc. (Incheon, Republic of Korea).

\section{Author contributions}

S.J.L., S.H.K., I.C., J.H.S., S.G.L., M.R.K., D.R.C., H.N.K. designed the study and contributed to in data analysis / interpretation. J.Y.K. and Y.R.J. collected data and contributed to data analysis / interpretation. An.S.-C., O.S., L.-L.P., Y.S.K., S.H.C, Ad.S-C. and J.S.E. collected data. All authors contributed to the preparation of the report, including critical review and revision of manuscript drafts, and approval of the final version.

\section{Competing interests}

M.G.I. received research support (paid to Northwestern University) from AiCuris, Janssen, and Shire; is a paid consultant for Adagio, AlloVir, Celltrion, Cidara, Genentech, Roche, Janssen, Shionogi, and Viracor Eurofins; and is also a paid member of Data and Safety Monitoring Boards for Janssen, Merck, SAB Biotherapeutics, Sequiris, Takeda, and Vitaeris. 
An.S.-C., O.S., and Ad.S.-C. have been investigators in COVID-19 clinical trials by Algernon

Pharmaceuticals, Atea Pharmaceuticals, Diffusion Pharmaceuticals, and Regeneron Pharmaceuticals, outside the scope of the submitted work, and by Celltrion, Inc., within the scope of the submitted work.

L.-L.P., Y.-S.K., and S.H.C. declare no conflicts.

J.S.E. has been an investigator in COVID-19 clinical trials by Enzychem Lifesciences, Bukwang Pharm.Co., Ltd, and SK chemicals outside the scope of the submitted work, and by Celltrion, Inc., within the scope of the submitted work.

J.Y.K. and Y.R.J. have been investigators in COVID-19 clinical trials by Daewoong Pharmaceuticals, Enzychem Lifesciences, and GC Pharma, outside the scope of the submitted work, and by Celltrion, Inc., within the scope of the submitted work.

S.J.L. and S.H.K. are employees of, and hold shares in, Celltrion, Inc. I.C., J.H.S., S.G.L., M.R.K., D.R.C., and H.N.K. are employees of Celltrion, Inc.

\section{References}

1 World Health Organization. Coronavirus disease (COVID-19) pandemic. https://www.euro.who.int/en/health-topics/health-emergencies/coronavirus-covid-19/novel-coronavirus2019-ncov (2020).

2 Pollard, C. A., Morran, M. P. \& Nestor-Kalinoski, A. L. The COVID-19 pandemic: a global health crisis. Physiol. Genomics 52, 549-557 (2020).

3 Johns Hopkins Coronavirus Resource Center. COVID-19 dashboard by the Center for Systems Science and Engineering (CSSE) at Johns Hopkins University (JHU).

https://coronavirus.jhu.edu/map.html (2021).

4 Eythorsson, E. et al. Clinical spectrum of coronavirus disease 2019 in Iceland: population based cohort study. BMJ 371, m4529 (2020).

5 Tabata, S. et al. Clinical characteristics of COVID-19 in 104 people with SARS-CoV-2 infection on the Diamond Princess cruise ship: a retrospective analysis. Lancet Infect. Dis. 20, 1043-1050 (2020).

6 World Health Organization. Maintaining essential health services: operational guidance for the COVID-19 context interim guidance. https://www.who.int/publications/i/item/WHO-2019-nCoV-essentialhealth-services-2020.1 (2020).

7 Liu, L. et al. Potent neutralizing antibodies against multiple epitopes on SARS-CoV-2 spike. Nature 584, 450-456 (2020). 
8 Shi, R. et al. A human neutralizing antibody targets the receptor-binding site of SARS-CoV-2. Nature 584, 120-124 (2020).

9 Wu, Y. et al. A noncompeting pair of human neutralizing antibodies block COVID-19 virus binding to its receptor ACE2. Science $368,1274-1278$ (2020).

10 Zost, S. J. et al. Potently neutralizing and protective human antibodies against SARS-CoV-2. Nature 584, 443-449 (2020).

11 Hoffmann, M. et al. SARS-CoV-2 cell entry depends on ACE2 and TMPRSS2 and is blocked by a clinically proven protease inhibitor. Cell 181, 271-280.e278 (2020).

12 Weinreich, D. M. et al. REGN-COV2, a neutralizing antibody cocktail, in outpatients with Covid-19. N. Engl. J. Med. 384, 238-251 (2021).

13 Chen, P. et al. SARS-CoV-2 neutralizing antibody LY-CoV555 in outpatients with Covid-19. N. Engl. J. Med. 384, 229-237 (2021).

14 Gottlieb, R. L. et al. Effect of bamlanivimab as monotherapy or in combination with etesevimab on viral load in patients with mild to moderate COVID-19: a randomized clinical trial. JAMA 325, 632-644 (2021).

15 ClinicalTrials.gov. A study of LY3819253 (LY-CoV555) and LY3832479 (LY-CoV016) in preventing SARS-CoV-2 infection and COVID-19 in nursing home residents and staff (BLAZE-2) (NCT04497987). https://clinicaltrials.gov/ct2/show/NCT04497987 (2021).

16 Lilly. Lilly's neutralizing antibody bamlanivimab (LY-CoV555) prevented COVID-19 at nursing homes in the BLAZE-2 trial, reducing risk by up to 80 percent for residents [press release] 2021).

$17 \mathrm{Kim}, \mathrm{C}$. et al. A therapeutic neutralizing antibody targeting receptor binding domain of SARS-CoV-2 spike protein. Nat. Commun. 12, 288 (2021).

$18 \mathrm{Kim}$, J. Y. et al. Safety, virologic efficacy, and pharmacokinetics of CT-P59, a monoclonal antibody against SARS-CoV-2 spike receptor binding protein: two randomised phase 1 studies in healthy subjects and patients with mild SARS-CoV-2 infection. In preparation (2021).

19 Bordi, L. et al. Rapid and sensitive detection of SARS-CoV-2 RNA using the Simplexa COVID-19 direct assay. J. Clin. Virol. 128, 104416 (2020).

20 Wolfel, R. et al. Virological assessment of hospitalized patients with COVID-2019. Nature 581, 465-469 (2020).

21 Xue, L. et al. Recommendations for the assessment and management of pre-existing drug-reactive antibodies during biotherapeutic development. AAPS J. 19, 1576-1586 (2017). 
22 Xue, L. \& Rup, B. Evaluation of pre-existing antibody presence as a risk factor for posttreatment anti-drug antibody induction: analysis of human clinical study data for multiple biotherapeutics. AAPS J. 15, 893-896 (2013).

23 Perkin, M. R. et al. Deaths in people from Black, Asian and minority ethnic communities from both COVID-19 and non-COVID causes in the first weeks of the pandemic in London: a hospital case note review. BMJ Open 10, e040638 (2020).

24 Wiley, Z. et al. Age, comorbid conditions, and racial disparities in COVID-19 outcomes. J. Racial Ethn. Health Disparities Jan 7 [Epub ahead of print], doi: 10.1007/s40615-40020-00934-40610 (2021).

25 Barek, M. A., Aziz, M. A. \& Islam, M. S. Impact of age, sex, comorbidities and clinical symptoms on the severity of COVID-19 cases: A meta-analysis with 55 studies and 10014 cases. Heliyon 6, e05684 (2020).

26 World Health Organization. Clinical management of COVID-19.

https://www.who.int/publications/i/item/clinical-management-of-covid-19 (2020).

\section{Tables}

Table 1. Baseline demographics and characteristics (ITT population) 


\begin{tabular}{|c|c|c|c|c|}
\hline & $\begin{array}{l}\text { CT-P59 } \\
40 \mathrm{mg} / \mathrm{kg} \\
n=105\end{array}$ & $\begin{array}{l}\text { CT-P59 } \\
80 \mathrm{mg} / \mathrm{kg} \\
n=111\end{array}$ & $\begin{array}{l}\text { CT-P59 } \\
\text { combined } \\
n=216\end{array}$ & $\begin{array}{l}\text { Placebo } \\
n=111\end{array}$ \\
\hline \multicolumn{5}{|l|}{ Age, years } \\
\hline \multirow{3}{*}{$\begin{array}{l}\text { Median (IQR) } \\
\geq 60, n(\%) \\
<60, n(\%)\end{array}$} & $\begin{array}{l}51.0(42 \\
60)\end{array}$ & $\begin{array}{l}51.0(40 \\
60)\end{array}$ & $\begin{array}{l}51.0(40 \\
60)\end{array}$ & $\begin{array}{l}52.0(41 \\
61)\end{array}$ \\
\hline & $27(25.7)$ & $28(25.2)$ & $161(74.5)$ & $30(27.0)$ \\
\hline & 78 (74.3) & $83(74.8)$ & $55(25.5)$ & $81(73.0)$ \\
\hline Male, $n(\%)$ & $59(56.2)$ & $59(53.2)$ & $98(45.4)$ & $48(43.2)$ \\
\hline \multicolumn{5}{|l|}{ Race, $n(\%)$} \\
\hline White & $94(89.5)$ & $96(86.5)$ & $190(88.0)$ & $96(86.5)$ \\
\hline Asian & $11(10.5)$ & $15(13.5)$ & $26(12.0)$ & $15(13.5)$ \\
\hline \multicolumn{5}{|l|}{ Ethnicity, $n(\%)$} \\
\hline Hispanic or Latino & $6(5.7)$ & $11(9.9)$ & $17(7.9)$ & $10(9.0)$ \\
\hline Non-Hispanic or non-Latino & $99(94.3)$ & $100(90.1)$ & $199(92.1)$ & $101(91.0)$ \\
\hline \multicolumn{5}{|l|}{ Region, $n(\%)$} \\
\hline USA & $1(1.0)$ & $4(3.6)$ & $5(2.3)$ & $3(2.7)$ \\
\hline Asia & $11(10.5)$ & $15(13.5)$ & $26(12.0)$ & $14(12.6)$ \\
\hline Europe & $93(88.6)$ & $92(82.9)$ & $185(85.6)$ & 94 (84.7) \\
\hline BMI, mean (SD), kg/m² & $27.1(4.8)$ & $27.1(4.1)$ & $27.1(4.5)$ & $26.8(4.2)$ \\
\hline \multicolumn{5}{|l|}{ Baseline comorbidities, $n(\%)$} \\
\hline Yes & $78(74.3)$ & $80(72.1)$ & $158(73.1)$ & $82(73.9)$ \\
\hline Confirmed SARS-CoV-2 infection ${ }^{\mathrm{a}}, n(\%)$ & $101(96.2)$ & $103(92.8)$ & $204(94.4)$ & $103(92.8)$ \\
\hline $\begin{array}{l}\text { Time since symptom onset, median (IQR), } \\
\text { days }\end{array}$ & $3.0(2,4)$ & $3.0(2,4)$ & $3.0(2,4)$ & $3.0(2,4)$ \\
\hline Moderate disease $^{\mathrm{b}}, n(\%)$ & $64(61.0)$ & $65(58.6)$ & $129(59.7)$ & $60(54.1)$ \\
\hline Received $\geq 1$ prior medication, $n(\%)$ & $19(18.1)$ & $23(20.7)$ & $42(19.4)$ & $26(23.4)$ \\
\hline
\end{tabular}




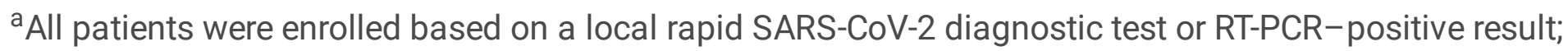
following enrollment, SARS-CoV-2 infection was confirmed centrally by RT-qPCR.

${ }^{b}$ Based on presence of $\mathrm{x}$-ray or computed tomography-confirmed pneumonia at screening.

$\mathrm{BMI}$, body mass index; IQR, interquartile range; ITT, intent to treat; RT-PCR, reverse transcription polymerase chain reaction; RT-qPCR, quantitative reverse transcription polymerase chain reaction; SARSCoV-2, severe acute respiratory syndrome coronavirus 2; SD, standard deviation.

Table 2. Efficacy endpoints (ITTI population) 


\section{Endpoint}

$\begin{array}{llll}\text { CT-P59 } & \text { CT-P59 } & \text { CT-P59 } & \text { Placebo } \\ 40 & 80 & \text { combined } & n=103 \\ \mathrm{mg} / \mathrm{kg} & \mathrm{mg} / \mathrm{kg} & n=204 & \\ n=101 & n=103 & & \end{array}$

\section{Primary endpoints}

Conversion to negative RT-qPCR to day 28

Negative titer threshold of $2.33 \log _{10}$ copies $/ \mathrm{ml}$

Median $(95 \% \mathrm{Cl})$ time to negative RT-qPCR, days

Improvement rate ratio $(95 \% \mathrm{Cl})$

$\begin{array}{llll}12.75 & 11.89 & 12.65 & 12.94 \\ (9.00- & (8.94- & (9.03- & (12.75- \\ 12.84) & 12.91) & 12.83) & 13.99) \\ 1.346 & 1.215 & 1.275 & - \\ (1.001- & (0.903- & (0.986- & \\ 1.810) & 1.634) & 1.649) & -\end{array}$

Negative titer threshold $3 \log _{10}$ copies $/ \mathrm{ml}^{\mathrm{b}}, n$

$\begin{array}{llll}0.048 & 0.198 & 0.063 & 101\end{array}$

Median $(95 \% \mathrm{Cl})$ time to negative RT-qPCR, days

Improvement rate ratio $(95 \% \mathrm{Cl})$

Negative titer threshold $4 \log _{10}$ copies $/ \mathrm{ml}^{\mathrm{b}}, n$

Median $(95 \% \mathrm{Cl})$ time to negative RT-qPCR, days

Improvement rate ratio $(95 \% \mathrm{Cl})$

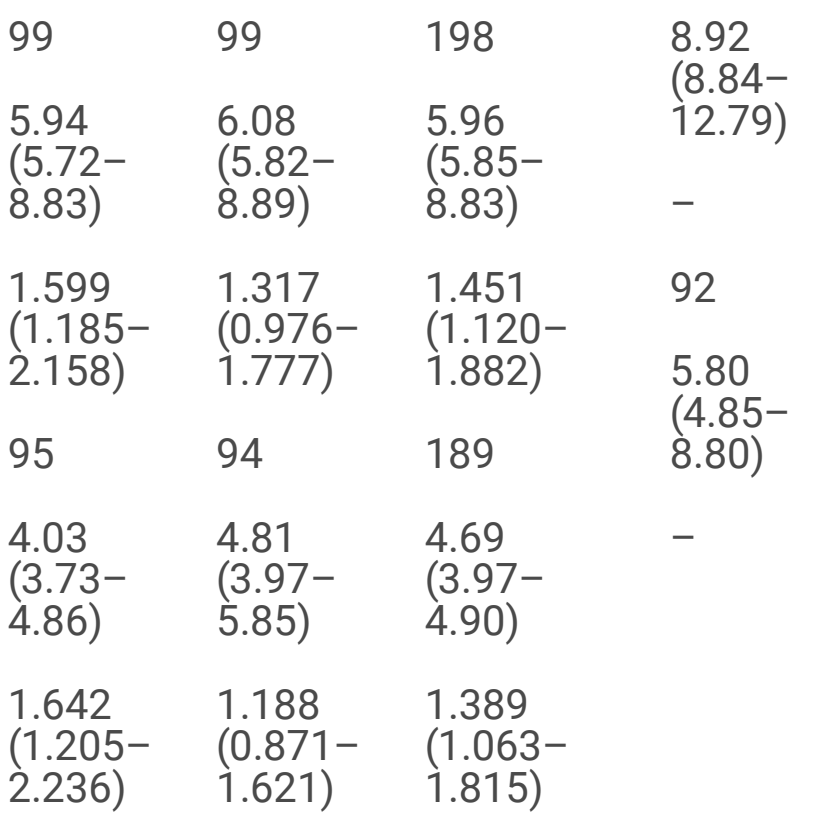

Clinical recovery to day 14

All patients

Median $(95 \% \mathrm{Cl})$ time to event, days

5.35

(3.97-

Clinical recovery ratio $(95 \% \mathrm{Cl})$

6.78)

6.23

(5.53-

5.72

(5.15-

7.85)

7.00)

8.77

$1562 \quad 1.429 \quad 1.489$

Log-rank test $P$-value ${ }^{a}$

(1.11-

(1.02-

2.20)

2.01)

(1.11-

2.01)

Mild SARS-CoV2 infection', $n$

$\begin{array}{llll}0.010 & 0.039 & 0.008 & 46\end{array}$

Median $(95 \% \mathrm{Cl})$ time to event, days

Clinical recovery ratio $(95 \% \mathrm{Cl})$

38

40

78

6.88

Moderate SARS-CoV2 infection ${ }^{c}, n$

$\begin{array}{lll}4.37 & 5.49 & 4.83 \\ (2.15- & (3.15- & (3.03-\end{array}$


Median $(95 \% \mathrm{Cl})$ time to event, days

Clinical recovery ratio $(95 \% \mathrm{Cl})$

Moderate SARS-CoV2 infection and aged $\geq 50$

years $^{\mathrm{b}, \mathrm{c}}, n$

Median $(95 \% \mathrm{Cl})$ time to event, days

Clinical recovery ratio $(95 \% \mathrm{Cl})$

\begin{tabular}{|c|c|c|c|}
\hline 7.67) & 7.60) & 5.92) & - \\
\hline $\begin{array}{l}1.512 \\
(0.90-\end{array}$ & $\begin{array}{l}1.741 \\
1.06-\end{array}$ & $\begin{array}{l}1.615 \\
(1.05-\end{array}$ & 57 \\
\hline 2.54$)$ & $2.86)$ & 2.49) & 10.81 \\
\hline 62 & 63 & 125 & n.c.) \\
\hline $\begin{array}{l}5.73 \\
(4.13- \\
7.33)\end{array}$ & $\begin{array}{l}7.30 \\
(5.58- \\
10.72)\end{array}$ & $\begin{array}{l}6.52 \\
(5.53- \\
7.69)\end{array}$ & $\begin{array}{l}- \\
38\end{array}$ \\
\hline $\begin{array}{l}1.689 \\
(1.06- \\
2.70)\end{array}$ & $\begin{array}{l}1.347 \\
(0.83- \\
2.18)\end{array}$ & $\begin{array}{l}1.511 \\
(0.99- \\
2.30)\end{array}$ & $\begin{array}{l}12.97 \\
\text { (6.81- } \\
\text { n.c.) }\end{array}$ \\
\hline 40 & 40 & 80 & - \\
\hline $\begin{array}{l}6.64 \\
(4.13- \\
11.94)\end{array}$ & $\begin{array}{l}7.29 \\
(5.54- \\
12.33)\end{array}$ & $\begin{array}{l}6.79 \\
(5.50- \\
10.72)\end{array}$ & \\
\hline $\begin{array}{l}1.584 \\
(0.86- \\
2.91)\end{array}$ & $\begin{array}{l}1.504 \\
(0.81- \\
2.78)\end{array}$ & $\begin{array}{l}1.545 \\
(0.90- \\
2.65)\end{array}$ & \\
\hline
\end{tabular}

\section{Secondary endpoints}

Patients with clinical symptoms requiring

hospitalization or oxygen therapy due to SARS-CoV-2

infection to day $28, n / N(\%)$

$\begin{array}{llll}4 / 101 & 5 / 103 & 9 / 204 & 9 / 103 \\ (4.0) & (4.9) & (4.4) & (8.7)\end{array}$

Mild SARS-CoV-2 infection ${ }^{c}$

Moderate SARS-CoV2 infection ${ }^{\mathrm{C}}$

$\begin{array}{llll}0 / 38 & 0 / 40 & 0 / 78 & 0 / 46\end{array}$

Moderate SARS-CoV2 infection aged $\geq 50$ years ${ }^{\mathrm{b}, \mathrm{c}}$

$\begin{array}{llll}4 / 62 & 5 / 63 & 9 / 125 & 9 / 57 \\ (6.5) & (7.9) & (7.2) & (15.8) \\ 3 / 40 & 4 / 40 & 7 / 80 & 9 / 38 \\ (7.5) & (10.0) & (8.8) & (23.7)\end{array}$

Patients achieving conversion to negative RT-qPCR (negative titer threshold: $2.33 \log _{10}$ copies $/ \mathrm{ml}$ ), $n / N$

(\%)

Up to day 14

Up to day 28

\begin{tabular}{|c|c|c|c|}
\hline $\begin{array}{l}68 / 101 \\
(67.3)\end{array}$ & $\begin{array}{l}68 / 103 \\
(66.0)\end{array}$ & $\begin{array}{l}136 / 204 \\
(66.7)\end{array}$ & $\begin{array}{l}62 / 103 \\
(60.2)\end{array}$ \\
\hline $\begin{array}{l}93 / 101 \\
(92.1)\end{array}$ & $\begin{array}{l}90 / 103 \\
(87.4)\end{array}$ & $\begin{array}{l}183 / 204 \\
(89.7)\end{array}$ & $\begin{array}{l}86 / 103 \\
(83.5)\end{array}$ \\
\hline $7(6.9)$ & $\begin{array}{l}11 \\
(10.7)\end{array}$ & $18(8.8)$ & $\begin{array}{l}15 \\
(14.6)\end{array}$ \\
\hline $4(4.0)$ & $5(4.9)$ & $9(4.4)$ & $9(8.7)$ \\
\hline $4(4.0)$ & $4(3.9)$ & $8(3.9)$ & 9 (8.7) \\
\hline
\end{tabular}




\begin{tabular}{|lllll|}
\hline Patients requiring mechanical ventilation, $n(\%)$ & 0 & $1(<1.0)$ & $1(<1.0)$ & 0 \\
\hline Patients requiring rescue therapy, $n(\%)$ & $7(6.9)$ & $\begin{array}{l}11 \\
(10.7)\end{array}$ & $18(8.8)$ & $\begin{array}{l}15 \\
\text { Patients requiring ICU transfer, } n(\%)\end{array}$ \\
\hline All-cause mortality, $n(\%)$ & 0 & 0 & 0 & 0 \\
\hline Patients achieving clinical recovery, $n / N(\%)$ & 0 & 0 & 0 & 0 \\
Up to day 7 & & & & \\
Up to day 14 & $53 / 95$ & $46 / 92$ & $99 / 187$ & $37 / 98$ \\
Up to day 28 & $(55.8)$ & $(50.0)$ & $(52.9)$ & $(37.8)$ \\
& $73 / 95$ & $72 / 92$ & $145 / 187$ & $62 / 98$ \\
& $(76.8)$ & $(78.3)$ & $(77.5)$ & $(63.3)$ \\
& $83 / 95$ & $79 / 92$ & $162 / 187$ & $70 / 98$ \\
& $(87.4)$ & $(85.9)$ & $(86.6)$ & $(71.4)$ \\
\hline
\end{tabular}

Table 3. Safety and tolerability (safety population)

\begin{tabular}{|c|c|c|c|}
\hline & $\begin{array}{l}\text { CT-P59 } \\
40 \mathrm{mg} / \mathrm{kg} \\
n=105\end{array}$ & $\begin{array}{l}\text { CT-P59 } \\
80 \mathrm{mg} / \mathrm{kg} \\
n=110\end{array}$ & $\begin{array}{l}\text { Placebo } \\
n=110\end{array}$ \\
\hline Any TEAE & $31(29.5)$ & $27(24.5)$ & $34(30.9)$ \\
\hline Related to study drug & $7(6.7)$ & $5(4.5)$ & $5(4.5)$ \\
\hline$\geq 1$ grade 3 TEAE $^{\mathrm{a}}$ & $5(4.8)$ & $4(3.6)$ & $2(1.8)$ \\
\hline Related to study drug & $1(1.0)$ & 0 & 0 \\
\hline Any TESAE & 0 & 0 & 0 \\
\hline Any TEAE leading to discontinuation & 0 & 0 & 0 \\
\hline \multicolumn{4}{|l|}{ Any TEAE of special interest } \\
\hline Infusion-related reactions & $1(1.0)$ & 0 & $2(1.8)$ \\
\hline Deaths & 0 & 0 & 0 \\
\hline
\end{tabular}

Data shown as $n(\%)$.

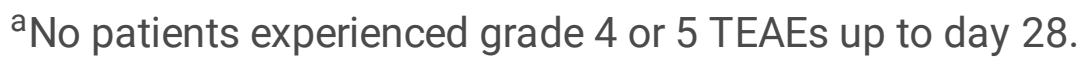

TEAE, treatment-emergent adverse event; TESAE, treatment-emergent serious adverse event.

\section{Figures}




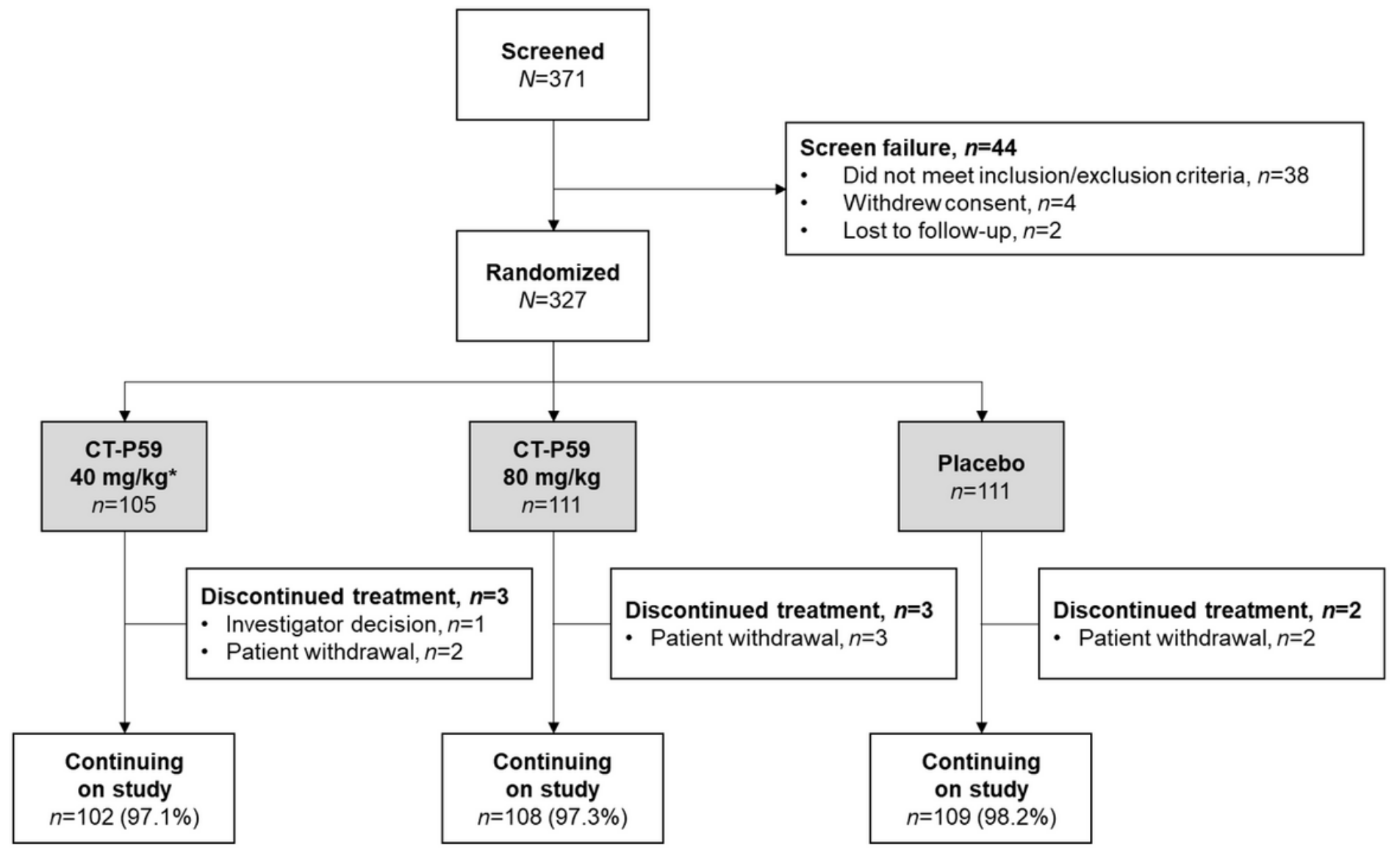

Figure 1

Patient disposition. *One patient was randomized to the placebo group but was administered study drug partially containing CT-P59; the individual was considered as having received CT-P59 $40 \mathrm{mg} / \mathrm{kg}$ and was excluded from the pharmacokinetic population. 

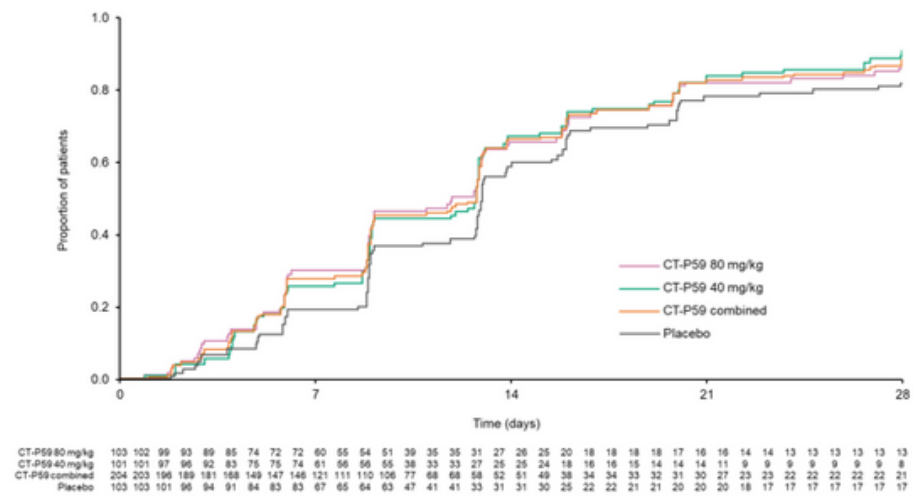

b

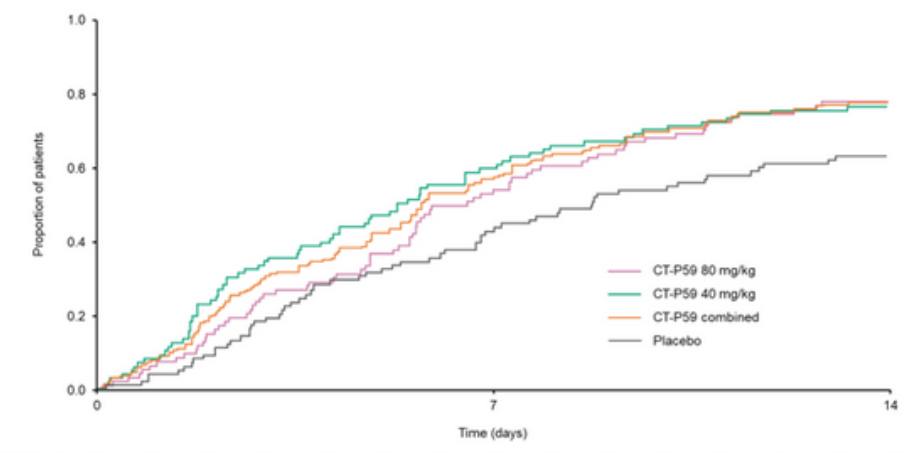

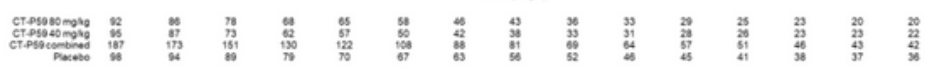

c

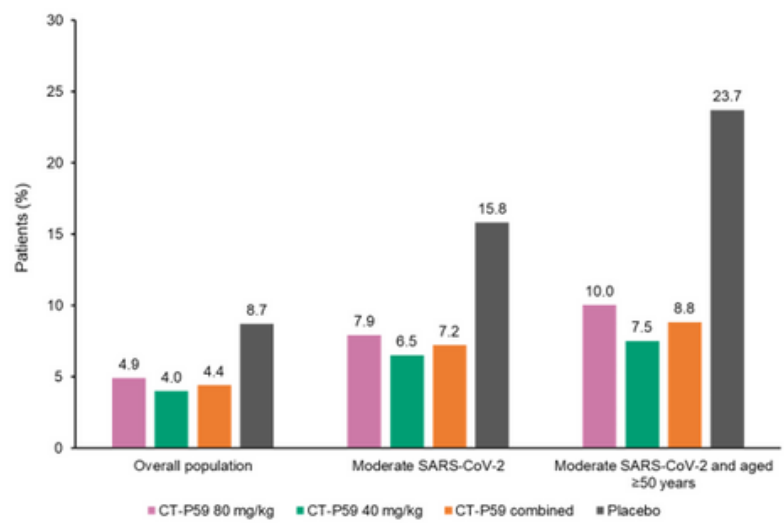

Figure 2

Efficacy endpoints (ITTI population). a, Kaplan-Meier plot of primary endpoint 'time to negative conversion' to day 28 (SARS-CoV-2-negative threshold: 2.33 log10 copies/ml). b, Kaplan-Meier plot of primary endpoint, time to clinical recovery to 14 days. c, Proportion of patients with clinical symptoms requiring hospitalization or oxygen therapy due to SARS-CoV-2 infection to day 28 in the overall 
population and subgroups by disease severity and age. ITTT, intent-to-treat infected; SARS-CoV-2, severe acute respiratory syndrome coronavirus 2 .

\section{Supplementary Files}

This is a list of supplementary files associated with this preprint. Click to download.

- CTP59Study3.2supplementaryinfo03Mar2021.docx

- CTP59AppendixCLEAN18Dec2020.pdf

- CTP59protocolXSAP.zip 